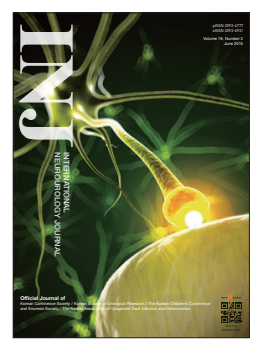

\title{
Does Methylphenidate Affect Cystometric Parameters in Spontaneously Hypertensive Rats?
}

\author{
Khae Hawn Kim¹, Ha Bum Jung', Don Kyoung Choi ${ }^{2}$, Geun Ho Park 3 , Sung Tae Cho² \\ ${ }^{1}$ Department of Urology, Gachon University Gil Medical Center, Gachon University College of Medicine, Incheon, Korea \\ ${ }^{2}$ Department of Urology, Hallym University Kangnam Sacred Heart Hospital, Hallym University College of Medicine, Seoul, Korea \\ ${ }^{3}$ Department of Pharmacology, Inha University College of Medicine, Incheon, Korea
}

Purpose: Methylphenidate (MPH) is one of the most commonly prescribed psychostimulants for attention deficit hyperactivity disorder (ADHD). However, there is limited research on its effects on lower urinary tract function. This study investigated changes in cystometric parameters after intragastric administration of MPH in conscious spontaneously hypertensive rats (SHRs), an animal model of ADHD.

Methods: Fourteen- to 16-week-old male SHRs ( $\mathrm{n}=10)$, weighing between 280 and $315 \mathrm{~g}$, were used. Three micturition cycles were recorded before administering MPH. One hour after each intragastric MPH injection, three cycles of cystometrogram were obtained in the awake condition. Various cystometric parameters were evaluated, including basal pressure (BP), maximal pressure (MP), threshold pressure (TP), bladder capacity (BC), micturition volume (MV), micturition interval (MI), and residual volume (RV). The data were analyzed using paired Student $t$-tests.

Results: Five SHRs were each administered a dose of 3-mg/kg MPH, and the other five received a dose of 6-mg $/ \mathrm{kg} \mathrm{MPH.} \mathrm{BP}$ and MP increased significantly in the rats that received the 3-mg/kg MPH injection, but not in those that received the 6-mg/ $\mathrm{kg}$ injection. BC, MV, and MI significantly increased in the rats that received the 6-mg/kg MPH injection, but not in those that received the 3-mg/kg injection. There were no significant changes in TP after either injection.

Conclusions: Significant increases in BC, MV, and MI after the 6-mg/kg MPH injection suggest that the peripheral and the central nervous systems may play important roles in bladder function in those receiving MPH for ADHD.

Keywords: Methylphenidate; Attention Deficit Disorder with Hyperactivity; Urodynamics; Rats

- Grant Support: This study was supported by Hallym University Research Fund 2013 (HURF-2013-23).

- Conflict of Interest: No potential conflict of interest relevant to this article was reported.

\section{INTRODUCTION}

Attention deficit hyperactivity disorder (ADHD) is one of the most commonly diagnosed and treated neurological behavior disorders in children and adolescents [1]. The prevalence of ADHD among children and adolescents is estimated to be 3\%$5 \%$, depending on the classification system used, with boys affected 3-10 times more frequently than girls [2,3]. ADHD is characterized by inattention, hyperactivity, impulsivity, or a combination of these symptoms, which compromise everyday, basic functions [1]. Although ADHD is the most commonly studied and diagnosed psychiatric disorder in children and adolescents, its cause remains unknown.

Methylphenidate (MPH) is a central nervous system (CNS) stimulant that has been widely prescribed for the treatment of $\mathrm{ADHD}$, and has been used for treating ADHD since more than

Corresponding author: Sung Tae Cho (iD http://orcid.org/0000-0002-4691-6159 Department of Urology, Hallym University Kangnam Sacred Heart Hospital, Hallym University College of Medicine, 1 Singil-ro, Yeongdeungpo-gu, Seoul 150-950, Korea

E-mail: cst326@paran.com / Tel: +82-2-829-5198 / Fax: +82-2-846-5198

Submitted: May 24, 2015 / Accepted after revision: May 31, 2015 
50 years [2]. It is presumed that the effects of $\mathrm{MPH}$ on $\mathrm{ADHD}$ symptoms are related to its role on dopaminergic and noradrenergic neurotransmission in the CNS [4].

Several studies have revealed co-occurrence of lower urinary tract (LUT) symptoms with enuresis and symptoms of ADHD $[5,6]$. Notably, the co-occurrence of enuresis and ADHD is approximately $30 \%$ [6]. However, little is known about the precise effects of MPH on the LUT. Only a limited number of studies have reported therapeutic effects of MPH in the treatment of giggle incontinence $[7,8]$. We recently performed a cystometric study about the effects of MPH in conscious mice that showed dose-dependent effects on the function of the LUT [9]. However, animal disease models are needed to establish the various effects of MPH on the LUT, particularly in patients with ADHD.

The spontaneously hypertensive rat (SHR) is the most extensively investigated genetic model and the only animal model that has been used to demonstrate all the behavioral characteristics of ADHD [10]. The aim of this study was to investigate whether intragastric injection of MPH affects cystometric parameters in conscious SHRs, an animal model for ADHD.

\section{MATERIALS AND METHODS}

\section{Animals}

Fourteen- to 16-week-old male SHRs $(\mathrm{n}=10)$, weighing between 280 and $315 \mathrm{~g}$, were purchased from Orient Bio Inc. (Seongnam, Korea). The animals were kept in standard housing facilities with a 12-hour light/dark cycle. The rats were given food and water were ad libitum. All experimental animals were handled according to the Guide for the Care and Use of Laboratory Animals of the National Institutes of Health.

\section{Surgical Procedures}

Each SHR was intraperitoneally anesthetized using xylazine (15 $\mathrm{mg} / \mathrm{kg}$ ) and ketamine (75 mg/kg). A suprapubic midline incision was made to expose the bladder and the urethra. Three days before the cystometric investigations, a surgery was performed for catheter placement to record intra-abdominal pressure (IAP) and intravesical pressure (IVP), and the procedure was based on a previously used method [11].

A polyethylene PE-50 catheter (Clay-Adams, Parsippany, NJ, USA) with a cuff was introduced into the bladder dome. A purse-string suture was applied to attach the catheter. A balloon was placed around the cuff proximal to the bladder for recording the IAP.
Another PE-50 catheter (Clay-Adams) with a cuff was introduced into the body of the stomach in a similar manner. All catheters were tunneled subcutaneously and transported percutaneously to the backs of the animals.

\section{Materials}

MPH (Ritalin, Novartis Pharmaceuticals, Basel, Switzerland) was dissolved in $0.9 \%$ saline solution. To evaluate the effects of two different doses of MPH ( $3 \mathrm{mg} / \mathrm{kg}$ and $6 \mathrm{mg} / \mathrm{kg})$, a 0.39 - to $0.94-\mathrm{mL}$ solution was administered intragastrically using the catheter.

\section{Cystometric Investigations}

Three days after the surgery, awake cystometry was performed. The catheter from the bladder was connected to a pressure transducer and an injection pump by using a T-tube. The other catheter to the balloon was connected to an extra pressure transducer for recording the IAP. A thin polyester sealing film prevented leakage through the connectors. These leakages can introduce errors while recording the IAP.

Micturition volumes (MVs) were measured using a collecting funnel connected to a force displacement transducer. Saline was continuously infused intravesically at a rate of $10 \mathrm{~mL} / \mathrm{hr}$. A cystometrogram was recorded using the AcqKnowledge software (Biopac Systems, Goleta, CA, USA) and an MP150 system (Biopac Systems) at a recording speed of $10 \mathrm{~mm} / \mathrm{min}$.

Three representative MVs were recorded consecutively to analyze the baseline data. One hour after each intragastric injection of MPH, cystometrograms were obtained consecutively using the same method. The results were compared with the corresponding baseline values.

The cystometric parameters were analyzed using the mean values of three consecutive micturition cycles. The parameters analyzed are listed as follows:

- Basal pressure (BP): the lowest bladder pressure during filling - Maximal pressure (MP): the maximum bladder pressure during micturition

- Threshold pressure (TP): the bladder pressure immediately before micturition

- Residual volume (RV): the volume of urine remaining after micturition

- Micturition volume (MV): the volume of urine passed

- Bladder capacity (BC: RV + MV)

- Micturition interval (MI): the intercontraction interval

IAP was defined as the recorded balloon pressure corrected 
by subtracting the lowest balloon pressure in each micturition cycle.

After the procedure, the animals were sacrificed by cervical dislocation. The bladder and urethra were excised and weighed.

\section{Statistical Analyses}

Data were analyzed using paired Student t-tests. GraphPad Prism (GraphPad Software Inc., La Jolla, CA, USA) was used for the same. The results were expressed as mean \pm standard er- ror. The level of significance was set at $\mathrm{P}<0.05$.

\section{RESULTS}

Five SHRs were each administered a low dose of MPH (3 mg/ $\mathrm{kg})$, and the other five received a higher dose of MPH (6 mg/ $\mathrm{kg})$. Body and bladder weights did not differ significantly between the two groups (Fig. 1).

The representative baseline cystometrograms and the cysto-

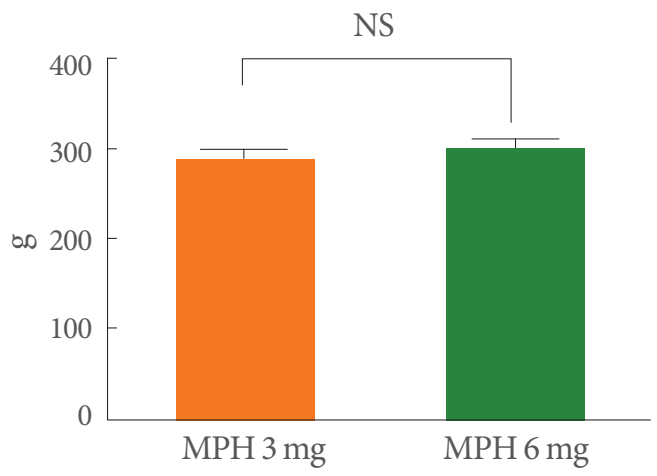

A

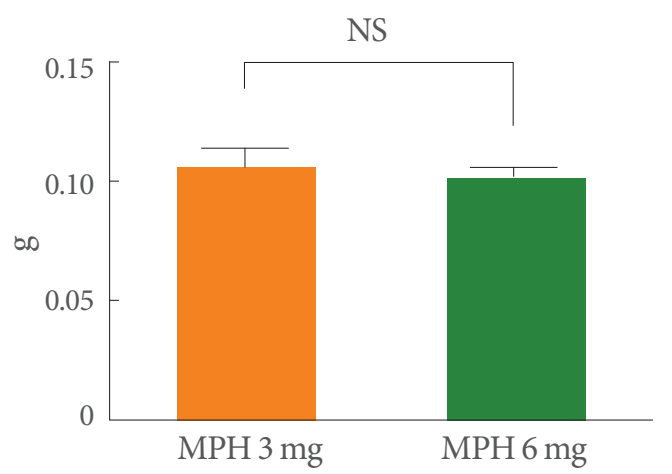

B

Fig. 1. Body weight (A) and bladder weight (B) in spontaneous hypertensive rats treated with 3- or 6-mg/kg MPH. There was no difference in body and bladder weights between the two groups. MPH, methylphenidate; NS, not significant.

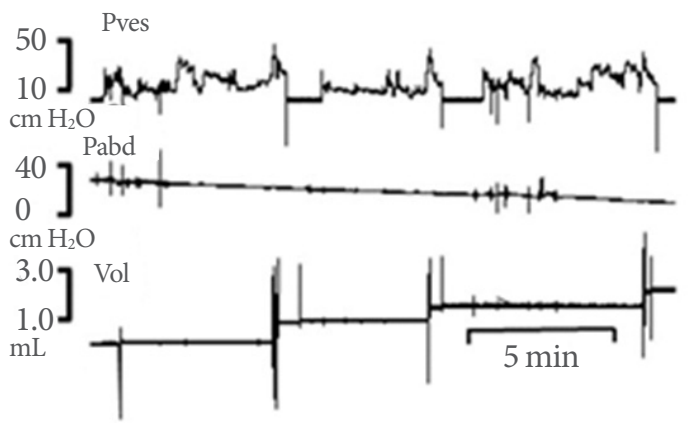

A
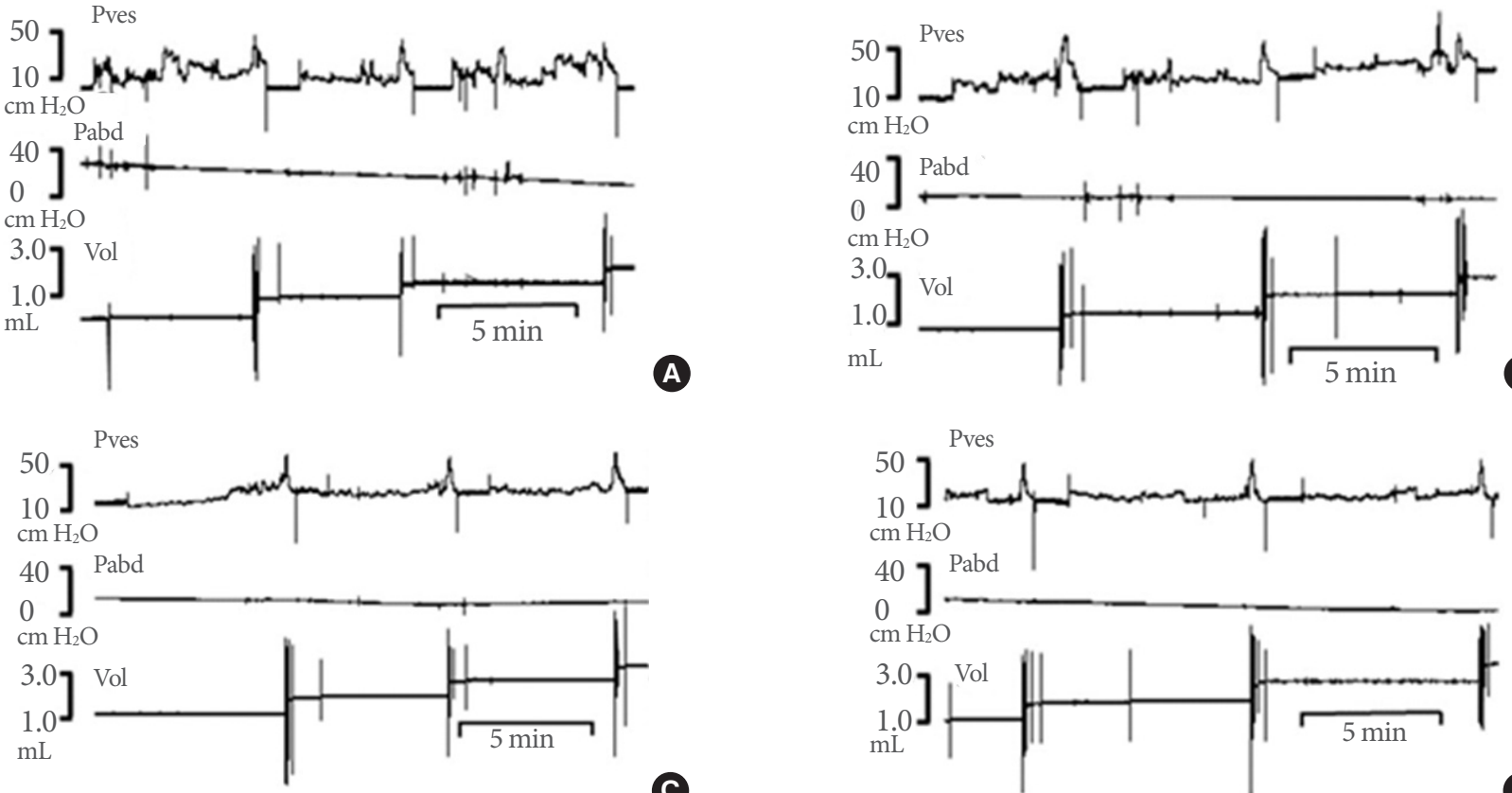

C

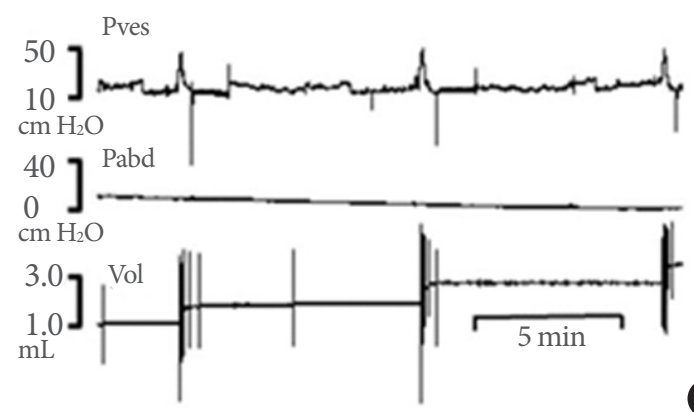

Fig. 2. Representative baseline cystometrograms and cystometrograms obtained 1 hour after intragastric injection of methylphenidate in the low-dose, 3-mg/kg group ( $\mathrm{A}$ and B, respectively) and in the high-dose, 6-mg/kg group (C and D, respectively). Pves, vesical pressure; Pabd, abdominal pressure; Vol, volume. 

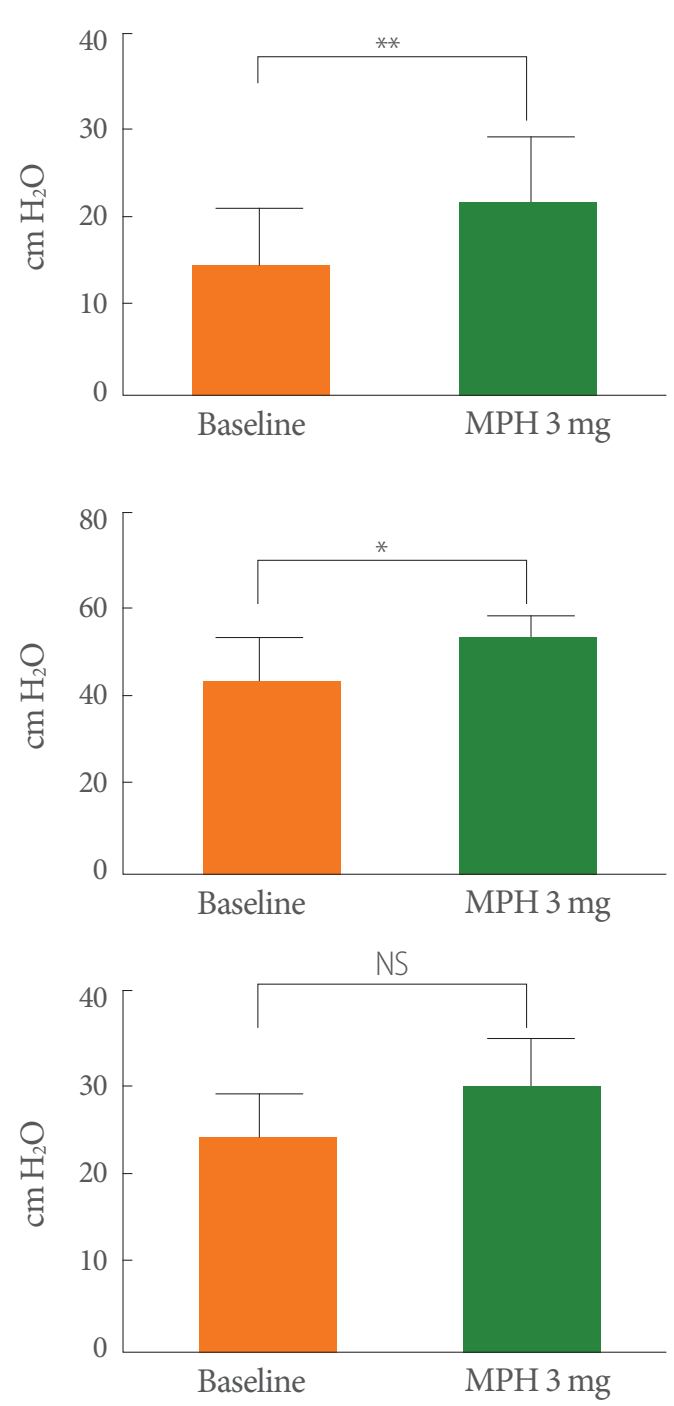
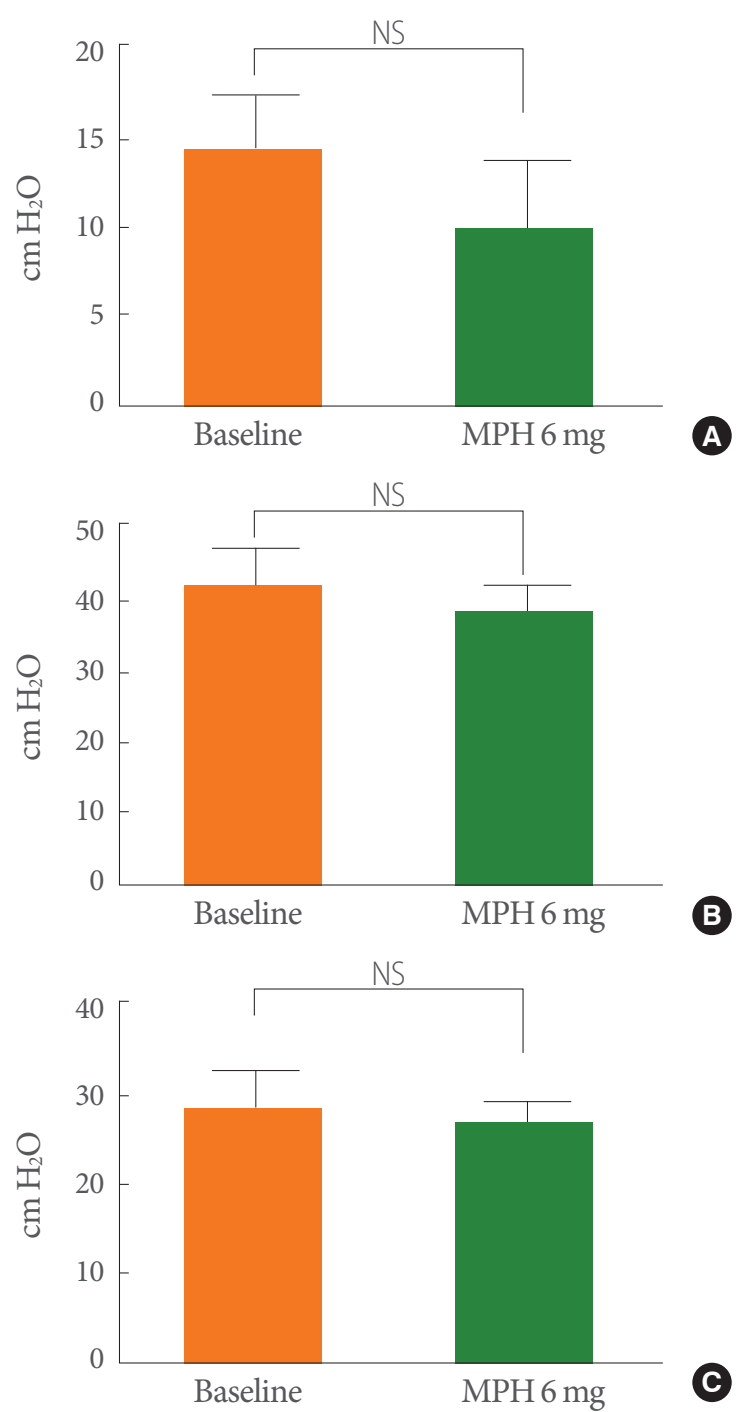

Fig. 3. Effects of each dose MPH on cystometric parameters. (A) Basal pressure. (B) Micturition pressure. (C) Threshold pressure. $\mathrm{MPH}$, methylphenidate; NS, not significant. ${ }^{\star} \mathrm{P}<0.05 .{ }^{* *} \mathrm{P}<0.01$.

metrograms obtained 1 hour after intragastric injections of $\mathrm{MPH}$ in the two groups are shown in Fig. 2. The different pressure parameters were compared on the basis of detrusor pressure, which is derived by subtracting IAP from IVP.

The BP and MP increased significantly after the 3-mg/kg MPH injection (BP: $13.9 \pm 6.9 \mathrm{~cm} \mathrm{H}_{2} \mathrm{O}$ before the injection vs. $23.2 \pm 6.9$ $\mathrm{cm} \mathrm{H}_{2} \mathrm{O}$ after the injection, $\mathrm{P}<0.01$; MP: $47.0 \pm 9.8 \mathrm{~cm} \mathrm{H}_{2} \mathrm{O}$ before vs. $57.9 \pm 8.2 \mathrm{~cm} \mathrm{H}_{2} \mathrm{O}$ after the injection, $\left.\mathrm{P}<0.05\right)$. However, $\mathrm{BP}$ and MP did not show any significant changes after the 6-mg/ $\mathrm{kg} \mathrm{MPH}$ injection. There were no significant changes in TP after either injection (Fig. 3).

Volume parameters, including $\mathrm{BC}, \mathrm{MV}$, and $\mathrm{MI}$ increased significantly after the 6-mg/kg MPH injection (BC: $0.47 \pm 0.07$
$\mathrm{mL}$ before the injection vs. $0.60 \pm 0.05 \mathrm{~mL}$ after the injection, $\mathrm{P}<0.01$; MV: $0.47 \pm 0.07 \mathrm{~mL}$ before vs. $0.60 \pm 0.05 \mathrm{~mL}$ after the injection, $\mathrm{P}<0.01$; MI: $4.68 \pm 0.21$ minutes before vs. $5.75 \pm 0.26$ minutes after the injection, $\mathrm{P}<0.01)$. However, there were no significant changes in these parameters after the 3-mg/kg MPH injection (Fig. 4). There was no RV at any time, either before or after the MPH injection, in any group.

\section{DISCUSSION}

This study investigated dose-dependent changes in cystometric parameters after intragastric administration of MPH in SHRs, an animal model of ADHD, and determined whether the CNS 

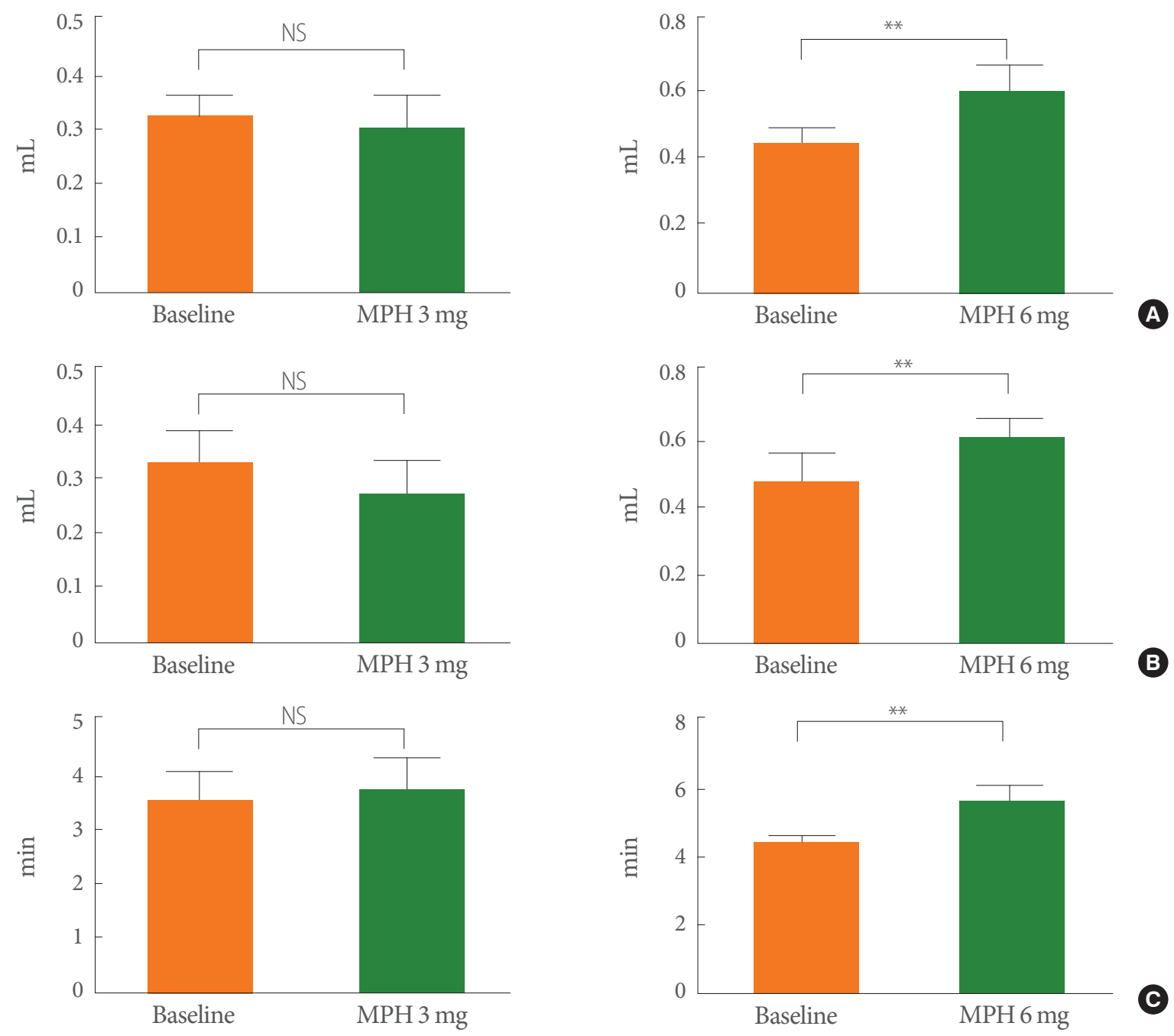

Fig. 4. Effects of each dose MPH on cystometric parameters. (A) Bladder capacity. (B) Micturition volume. (C) Micturition interval. $\mathrm{MPH}$, methylphenidate; NS, not significant. ${ }^{*} \mathrm{P}<0.01$.

stimulant MPH could influence LUT function in this animal disease model.

The SHR is a well validated and frequently used animal model of ADHD [10,11]. It has been reported that although other strains and species may present hyperactivity and attention deficits in accordance with the genetic, environmental, and pharmacological interventions, the SHR is the only strain to have the main behavioral appearances of ADHD [11,12]. In addition, because of their multiple, coexisting phenotypic characteristics, SHRs have also been used as models of other diseases, such as hypertension, stroke, anxiety, and detrusor overactivity $[13,14]$. Therefore, in this study, we evaluated the cystometric response to MPH by using conscious SHRs.

Previous studies reported increased afferent sensitivity in
SHRs that may contribute to their bladder hyperactivity [15]. SHRs show increased voiding frequency, and conversely, decreased BC and MV compared to Wistar rats [13]. The results of our study, using the animal model of ADHD, are similar to those of previous studies on SHRs.

$\mathrm{MPH}$ is a CNS stimulant that has been used as the first choice in the treatment of ADHD in children [2,4]. $\mathrm{MPH}$ blocks the reuptake of dopamine and noradrenaline, but its precise mechanisms of action on symptoms of ADHD are not clear [12]. MPH has also been used in the treatment of narcolepsy, mild depression, brain injury, cancer, cognitive disorders, and in combination with other drugs, in the treatment of chronic pain [16]. Side effects are usually mild and are generally well tolerated by patients. The most common side effects are in- 
somnia, stomachache, headache, and anorexia [17]. Usually, these side effects either diminish in severity over time to tolerable levels or can be managed effectively during the course of the treatment $[16,17]$.

In this study, we used two different doses of MPH: $3 \mathrm{mg} / \mathrm{kg}$ and $6 \mathrm{mg} / \mathrm{kg}$. Most of the previous studies have used significantly higher doses $(2-15 \mathrm{mg} / \mathrm{kg}$, intravenously or $10-50 \mathrm{mg} /$ $\mathrm{kg}$, intraperitoneally) than those used clinically in humans $(0.3-1 \mathrm{mg} / \mathrm{kg})$ [18]. In SHRs, the effects of MPH over a wide range of doses $(0.1-20 \mathrm{mg} / \mathrm{kg}$, intraperitoneally) have been evaluated [19,20]. Intraperitoneal MPH $(5 \mathrm{mg} / \mathrm{kg}$ and $10 \mathrm{mg} /$ $\mathrm{kg}$ ) was approximately twice as potent as intragastric MPH regarding the increase of extracellular dopamine levels and locomotion stimulation [18]. MPH doses used in this present study were selected based on a previous study.

In our previous study, we administered a low dose of MPH $(1.25 \mathrm{mg} / \mathrm{kg})$ as well as high doses of MPH $(2.5 \mathrm{mg} / \mathrm{kg}$ and 5.0 $\mathrm{mg} / \mathrm{kg}$ ) in mice by using a gastric catheter. We observed typical micturition cycles of mice using awake cystometry. MP decreased and BC increased without any elevation in RV in the low-dose group. However, in the high-dose groups, we could not find any typical configurations of micturition cycles, except urine leakage [9].

Unlike our previous mice study, this study showed typical micturition cycles, regardless of the dose of MPH for all SHRs. With high doses of MPH $(6 \mathrm{mg} / \mathrm{kg})$, the SHRs showed increased MV, MI, and BC with no increase in RV. However, with low doses of MPH (3 mg/kg), the SHRs showed no significant changes in MV, MI, or BC. These findings suggest that despite being a CNS stimulant, MPH may play a pivotal role in the peripheral nervous system (PNS) of the LUT in patients receiving treatment for ADHD with high doses of MPH (6 mg/kg). To date, the exact oral dose of MPH to regulate LUT has not been established, especially for SHRs. However, our results provide knowledge on the doses of MPH to regulate LUT involving PNS action while maintaining CNS effects.

One of the limitations of this study was the absence of a control group to compare the effects of MPH on the LUT. A control group consisting of normal Sprague-Dawley or Wistar rats should have been included to compare the results of the effects of MPH on a rat model without ADHD. Another limitation is the lack of morphological assessment using molecular assays. We only evaluated the functional role of MPH in the LUT by using awake rat cystometry. However, the combined use of functional and molecular assays would have elucidated the pre- cise effects of MPH on the LUT of SHRs. Despite these limitations, this study provides important information on the effects of MPH on the LUT.

In conclusion, intragastric injection of MPH $(6 \mathrm{mg} / \mathrm{kg})$ in SHRs showed significant increases in BC, MV, and MI. This suggests that the PNS, as well as the CNS, may have important roles in LUT function when treating ADHD with MPH.

\section{REFERENCES}

1. Feldman HM, Reiff MI. Clinical practice: attention deficit-hyperactivity disorder in children and adolescents. N Engl J Med 2014;370:838-46.

2. Storebo OJ, Rosendal S, Skoog M, Groth C, Bille T, Rasmussen KB, et al. Methylphenidate for attention deficit hyperactivity disorder (ADHD) in children and adolescents. Cochrane Database Syst Rev 2012;5:CD009885.

3. Kaye JD, Palmer LS. Characterization and management of voiding dysfunction in children with attention deficit hyperactivity disorder. Urology 2010;76:220-4.

4. Engert V, Pruessner JC. Dopaminergic and noradrenergic contributions to functionality in ADHD: the role of methylphenidate. Curr Neuropharmacol 2008;6:322-8.

5. Yang TK, Guo YJ, Chen SC, Chang HC, Yang HJ, Huang KH. Correlation between symptoms of voiding dysfunction and attention deficit disorder with hyperactivity in children with lower urinary tract symptoms. J Urol 2012;187:656-61.

6. Burgu B, Aydogdu O, Gurkan K, Uslu R, Soygur T. Lower urinary tract conditions in children with attention deficit hyperactivity disorder: correlation of symptoms based on validated scoring systems. J Urol 2011;185:663-8.

7. Berry AK, Zderic S, Carr M. Methylphenidate for giggle incontinence. J Urol 2009;182(4 Suppl):2028-32.

8. Chang JH, Lee KY, Kim TB, Yoon SJ, Lee T, Kim KH. Clinical and urodynamic effect of methylphenidate for the treatment of giggle incontinence (enuresis risoria). Neurourol Urodyn 2011;30:133842.

9. Choi SH, Cho YS, Cho ST, Lee T, Kim KH. Acute dose-related differential effects of methylphenidate on murine cystometric parameters. Int Neurourol J 2013;17:162-7.

10. Russell VA. Neurobiology of animal models of attention-deficit hyperactivity disorder. J Neurosci Methods 2007;161:185-98.

11. Sagvolden T. Behavioral validation of the spontaneously hypertensive rat (SHR) as an animal model of attention-deficit/hyperactivity disorder (AD/HD). Neurosci Biobehav Rev 2000;24:31-9. 
12. Kishikawa Y, Kawahara Y, Yamada M, Kaneko F, Kawahara H, Nishi A. The spontaneously hypertensive rat/Izm (SHR/Izm) shows attention deficit/hyperactivity disorder-like behaviors but without impulsive behavior: therapeutic implications of low-dose methylphenidate. Behav Brain Res 2014;274:235-42.

13. Shimizu S, Saito M, Oiwa H, Ohmasa F, Tsounapi P, Oikawa R, et al. Olmesartan ameliorates urinary dysfunction in the spontaneously hypertensive rat via recovering bladder blood flow and decreasing oxidative stress. Neurourol Urodyn 2014;33:350-7.

14. Jin LH, Andersson KE, Kwon YH, Yoon SM, Lee T. Selection of a control rat for conscious spontaneous hypertensive rats in studies of detrusor overactivity on the basis of measurement of intra-abdominal pressures. Neurourol Urodyn 2010;29:1338-43.

15. Clouse AK, Jugus MJ, Eisennagel SH, Laping NJ, Westfall TD, Thorneloe KS. Voltage-gated $\mathrm{Na}+$ channel blockers reduce functional bladder capacity in the conscious spontaneously hypertensive rat. Urology 2012;79:1410.e1-6.

16. Morton WA, Stockton GG. Methylphenidate abuse and psychiatric side effects. Prim Care Companion J Clin Psychiatry 2000;2:15964.

17. Firestone P, Musten LM, Pisterman S, Mercer J, Bennett S. Shortterm side effects of stimulant medication are increased in preschool children with attention-deficit/hyperactivity disorder: a doubleblind placebo-controlled study. J Child Adolesc Psychopharmacol 1998;8:13-25.

18. Gerasimov MR, Franceschi M, Volkow ND, Gifford A, Gatley SJ, Marsteller D, et al. Comparison between intraperitoneal and oral methylphenidate administration: a microdialysis and locomotor activity study. J Pharmacol Exp Ther 2000;295:51-7.

19. Carmack SA, Howell KK, Rasaei K, Reas ET, Anagnostaras SG. Animal model of methylphenidate's long-term memory-enhancing effects. Learn Mem 2014;21:82-9.

20. Heal DJ, Smith SL, Kulkarni RS, Rowley HL. New perspectives from microdialysis studies in freely-moving, spontaneously hypertensive rats on the pharmacology of drugs for the treatment of ADHD. Pharmacol Biochem Behav 2008;90:184-97. 